\title{
Differential effect of epithelial cell-conditioned medium fractions on preimplantation mouse embryo development
}

\section{Greet Leppens ${ }^{1}$ and Denny Sakkas}

Clinic of Sterility, Department of Obstetrics and Gynaecology, Hospital Cantonal University of Geneva, 1211 Geneva 14, Switzerland

'To whom correspondence should be addressed at: Laboratoires des Gamètes, Policlinique de Stérilité, Hôpital Cantonal Universitaire de Genève, 20 rue Alcide-Jentzer, 1211 Genève 14, Switzerland

Coculture studies using preimplantation embryos have led to a number of conflicting studies. In the human, ethical considerations have led to the preferential use of epithelial cell lines as distinct from human Fallopian tube cells. In an attempt to isolate factors influencing embryo development we have cultured 2-cell OF1 mouse embryos in media [Ménézo's B2 and Whittingham's T6 supplemented with vitamins and amino acids (T6VA)] conditioned on two types of kidney epithelial cells (MBDK and Vero). Different molecular weight fractions of conditioned medium were used to show the absence or presence of specific embryotrophic factors. With MDBK cells, $\mathrm{B2}$ conditioned medium enhanced embryo development up to the blastocyst stage, while no blastocysts developed in $\mathrm{B} 2$ alone. When using T6VA medium, both the control and conditioned media showed a high percentage of blastocyst formation (57.0 and 54.0\% respectively), while the different molecular weight fractions showed no added improvement. With Vero cells, B2 alone, B2 conditioned medium and fractions were all detrimental to embryo development. A high percentage of blastocyst formation (between 64.7 and 75.8\%) was observed in T6VA alone, T6VA conditioned medium and fractions. Low blastocyst formation in a control medium can show strong positive results when medium is conditioned by cells. In contrast, a good base medium, such as T6VA, can equal the results using conditioned medium. Different cells in contact with different types of medium show variability in the pattern of responses, highlighting the presence of false positives in coculture studies.

Key words: coculture/conditioned medium/embryo culture

\section{Introduction}

A number of problems are present during the in-vitro culture of preimplantation embryos compared to their in-vivo counterparts. In particular, embryos experience a delay in development of up to $24 \mathrm{~h}$ and a decrease in viability (Harlow and Quinn, 1982; Sakkas et al., 1989a,b). Furthermore, species-specific blocks have been shown in cultured embryos, a phenomenon which appears to be linked to embryonic genome activation.
In the mouse this occurs in outbred strains at the 2-cell stage (Goddard and Pratt, 1983).

For a number of years, several groups have attempted to improve embryo development in vitro and surpass cleavage arrest by culturing embryos in different media. This has varied from using simple chemically defined media to more complex media (Quinn et al., 1982; Chatot et al., 1989; Gardner and Sakkas, 1993). Energy substrates (pyruvate, lactate and glucose) have also been shown to be important. For example, Brown and Whittingham (1991) showed that the presence of energy sources could be changed at different stages of embryo development, showing that pyruvate and lactate were useful to pass the 2-cell block while glucose was necessary to reach the blastocyst stage.

In contrast to using media of known composition, many laboratories have proposed that feeder cells could contribute to the improvement of embryo development by changing the metabolite pool in the vicinity of the embryo. In 1987, Gandolfi and Moor successfully used coculture with sheep oviduct epithelial cells to improve the culture and viability of sheep embryos. Similar studies were subsequently conducted by Eyestone and First (1989) in cattle, Ouhibi et al. (1990) and Sakkas and Trounson (1990) in mice and Sakkas et al. (1989b) in goats. In the human, coculture using Fallopian tube cells (Bongso et al., 1989; Yeung et al., 1992) and bovine uterine fibroblast cells (Wiemer et al., 1989) has been reported to improve pregnancy rates; however, the use of controlled epithelial cell lines is more ethically acceptable due to the possibility of introducing viruses and infecting the patient's embryo.

An extension of the coculture studies is the investigation of embryo development in conditioned medium. Eyestone and First (1989) examined the effect on cattle embryos of coculture and conditioned medium from oviductal cells in M199 with $10 \%$ fetal calf serum (FCS). They showed an enhancement in embryo development until the blastocyst stage in medium which had been in contact with cells, i.e. embryo development in coculture and conditioned medium was $22 \%$ compared to $3 \%$ in control medium. Ellington et al. (1990) and Mermillod et al. (1993) also examined the use of conditioned medium to improve cattle embryo culture and reported a low molecular weight molecule which enhanced embryo development when medium (M199) was conditioned on oviductal cells.

Unfortunately, the true effect of coculture remains ambiguous. Although some authors suggest that coculture can have positive and negative effects (Bavister, 1992), others argue more positively for the presence of embryotrophic factors (Bongso et al., 1993). In this study, we have attempted to 
rectify this ambiguity by searching for specific factors in cellconditioned medium which may enhance embryo development.

The aim of this work was to show if media conditioned by Madin-Darby bovine kidney (MDBK) or green monkey kidney (Vero) epithelial cells could enhance embryo development. In this study we compared mouse embryo development in two different base media (Ménézo's B2 and Whittingham's T6 supplemented with vitamins and amino acids) conditioned with epithelial cells. Different molecular weight fractions of conditioned media were used to determine the presence of specific embryotrophic factors.

\section{Materials and methods}

\section{Embryo culture}

Embryos were obtained from 3-4 week old, outbred (OF1) mice (BRL, Fullinsdorf, Switzerland) which exhibit the 2-cell block in vitro (Sakkas et al., 1993). The females underwent ovulation induction by i.p. injection of 5 IU pregnant mare's serum (PMS) (Folligon; Veterinaria, Zurich, Switzerland) and an additional injection $48 \mathrm{~h}$ later of 5 IU human chorionic gonadotrophin (HCG, Chorulon; Veterinaria). They were then placed individually with C57/BL males of proven fertility. The following morning, the effectiveness of mating was confirmed by the presence of a vaginal plug (day 1). The 2-cell embryos were collected $43-45 \mathrm{~h}$ post-HCG by flushing the oviduct in HEPES-buffered medium (M2) (Whittingham, 1971). A pool of 2-cell embryos was collected before each experiment. For each treatment tested, three drops of $20 \mu \mathrm{l}$ of media were prepared under light white mineral oil (Sigma Pharmaceuticals, Buchs, Switzerland) at $37^{\circ} \mathrm{C}$ in $5 \% \mathrm{CO}_{2}$ in air. The 2-cell embryos were washed twice and then transferred to the last drop for culture.

\section{In-vitro fertilization experiments}

Spermatozoa were collected from 10-16 week old OFI males by squeezing the vas deferens and cauda epididymis in T6 medium containing $15 \mathrm{mg} / \mathrm{ml}$ bovine serum albumin (BSA; Sigma). Oocytes were collected by tearing the ampulla to release the oocyte-cumulus complex into the sperm suspension. In-vitro fertilization (IVF) was performed between 08.30 and 09.30 in the morning. After $8 \mathrm{~h}$, in-vitro fertilized embryos were transferred into Whittingham's T6 medium containing $4 \mathrm{mg} / \mathrm{ml} \mathrm{BSA} \mathrm{(T6)} \mathrm{for} \mathrm{a} \mathrm{period} \mathrm{of} 14 \mathrm{~h}$ until the early 2-cell stage. At this stage, embryos were placed in $20 \mu \mathrm{l}$ drops of the different types of media being examined: B2 conditioned medium and fractions and T6VA conditioned medium without BSA and fractions.

\section{Culture media}

During these experiments, two types of media were compared, Ménézo's B2 (pH 7.2-7.5, osmolarity 275-305 mosmol $/ \mathrm{kg}$; BioMerieux SA, 69280 Marcy, l'Etoile, France), which is often used for human IVF, and Whittingham's T6 ( $\mathrm{pH} \mathrm{7.2-}$ 7.5, osmolarity 280-290 mosmol/kg; Quinn et al., 1982). T6 medium was supplemented with amino acids and vitamins used at the concentration present in Eagle's minimal essential medium (Gibco, Basel, Switzerland), L-glutamine (4.0 mM) and glycine $(0.40 \mathrm{mM})$ (T6VA); no BSA was added to this medium. The composition of this medium has been described previously (Sakkas et al., 1994). Antibiotics were added to all culture media (100 IU penicillin G, $1000 \mu \mathrm{g} / \mathrm{ml}$ streptomycin sulphate).

\section{Preparation of conditioned medium and fractions}

For these experiments, MDBK and Vero cells were used to condition medium. These cells have previously been reported to improve embryo development (Ouhibi et al., 1990; Lai et al., 1992) and share the same embryonic origin (mesoderm) with oviduct cells. To maintain the cell lines we used Dulbecco's modified Eagle's medium (DMEM; Gibco) with $10 \%$ FCS. Before each experiment a passage of the MDBK or Vero cells was performed (at the same time as the PMS injection) and $0.3 \times 10^{6}$ cells $/ \mathrm{ml}$ were inseminated in a flask. At $45 \mathrm{~h}$ before embryo collection (at the same time as the HCG injection), $5 \mathrm{ml}$ of DMEM with $10 \%$ FCS was removed and the monolayer of cells was covered by $2.5 \mathrm{ml}$ of B2 or T6VA medium. Then, $6 \mathrm{~h}$ before collecting the 2-cell embryos, the conditioned medium (CM-B2 or CM-T6VA) was taken off. Some of this CM was filtered through Millipore membranes of different pore sizes: $10,30,100,300 \mathrm{kDa}$ (UltrafreeMC polysulfone; Millipore, Volketswil, Switzerland). Medium passing through the filters was taken. We obtained six different treatment groups: four different fractions containing conditioned media proteins with molecular weights of $<10,30,100$ and $300 \mathrm{kDa}$, total conditioned medium (CM), which was not filtered through any membrane but centrifuged to remove cells, and the control medium. The different treatment groups obtained $(10,30,100,300 \mathrm{kDa}$ CM-B2 or CM-T6VA and B2 or T6VA respectively) were placed in $20 \mu \mathrm{l}$ drops under oil.

The following numbers of replicates were performed for each experiment: CM-B2 conditioned on MDBK cells (14 replicates), CM-T6VA conditioned on MDBK cells (12 replicates), CM-B2 conditioned on Vero cells (seven replicates), CM-T6VA conditioned on Vero cells (nine replicates). For all the experiments, 5-10 2-cell embryos were placed in each drop. Embryos were pooled to allow sufficient numbers to be tested, as six different culture conditions were being assessed for each experiment.

\section{Embryo assessment and cell number determination}

Embryo cell number was determined at 72,96 and $120 \mathrm{~h}$ after HCG when embryos should be at the 4- or 8-cell, morula and blastocyst stage respectively. To assess the cell numbers of morulae and blastocysts, a modification of Tarkowski's airdrying technique was used (Tarkowski, 1966). Briefly, embryos were placed in $0.5 \%$ sodium citrate for 3-4 min, transferred on a glass slide and fixed using glacial acetic acid:ethanol $(1: 3 \mathrm{v} / \mathrm{v})$. Blastomere nuclei were subsequently stained with $10 \%$ Giemsa.

\section{Statistics}

The $\chi^{2}$ test was used to compare the percentages of embryos which reached different morphological stages: cleavage, morula, blastocyst. Multiple comparisons were performed using the Bonferroni procedure. Differences between the mean 
number of cells in the embryos at the blastocyst stage were compared using one-way analysis of variance and Scheffe's $F$-test for multiple comparisons.

\section{Results}

\section{Embryo development from the 2-cell stage in different media conditioned by $\mathrm{MDBK}$ cells}

\section{Ménézo's B2}

In B2 alone there was no blastocyst development. Only seven out of 82 reached the morula stage. Blastocyst formation was observed in all media which had been in contact with cells. The highest percentage of embryos $(35.6 \%)$ developing to the blastocyst stage was observed in fraction $300 \mathrm{kDa}$ (Figure 1A); however, this failed to be significantly different from the other fractions. As this beneficial effect was not observed in the other fractions, it may suggest that a substance having a molecular mass of between 100 and $300 \mathrm{kDa}$ may have enhanced embryo development. When comparing cell numbers using Scheffe's $F$-test, no culture group differed statistically from the others (Figure $1 \mathrm{~A}$ ).

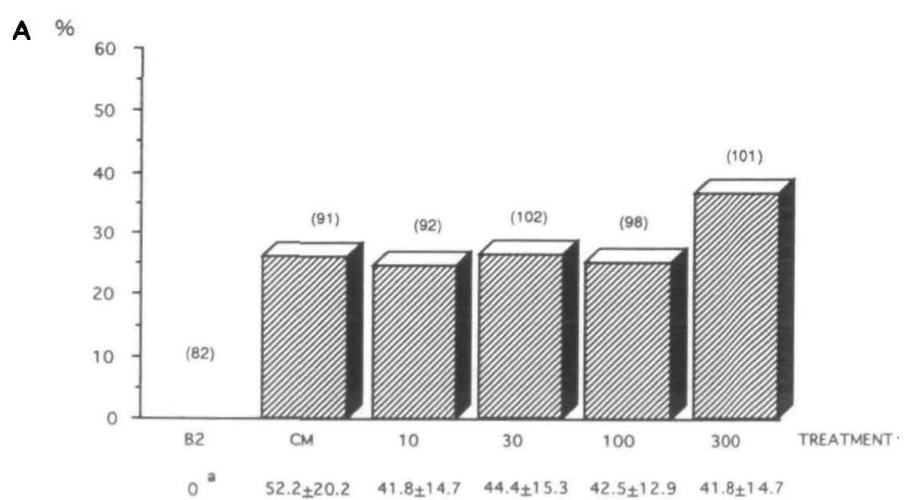

B

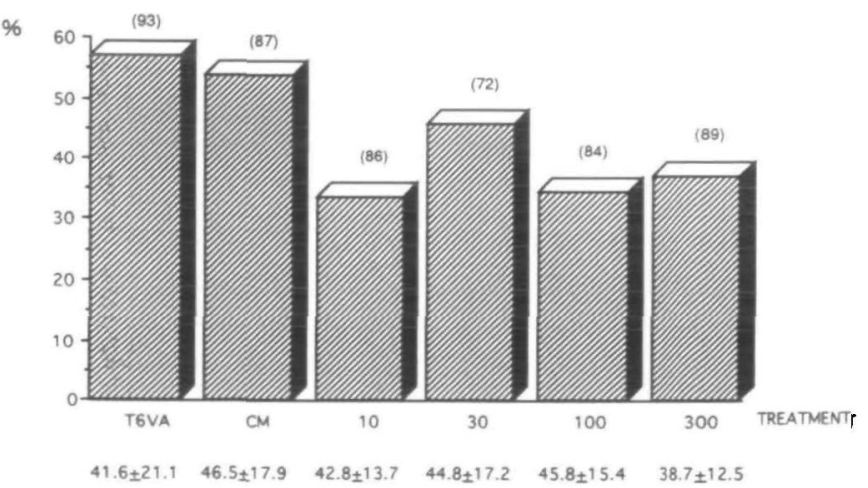

Figure 1. The percentage of 2-cell embryos developing to the blastocyst stage $120 \mathrm{~h}$ post-HCG on MDBK cells (A) in B2, conditioned medium (CM-B2) and fractions $(<10,30,100$ and $300 \mathrm{kDa}$ ), and (B) in T6VA medium, conditioned medium (CM-T6VA) and fractions. Numbers of embryos observed are in parentheses. Mean cell numbers \pm SD of blastocysts are shown

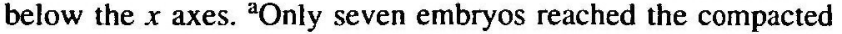
stage.

\section{T6VA medium}

In the light of the results obtained using B2, we chose to use a medium that was more suited to mouse embryo development, an approach that we have used previously (Sakkas et al., 1989a; Sakkas and Trounson, 1990). The modification of the T6 we have used (T6VA) supported development of 2-cell mouse embryos to the hatching blastocyst stage (unpublished data). When T6VA was conditioned (CM-T6VA), a high percentage of blastocyst development was observed, but this was not different to that in T6VA alone, 57.0 and $54.0 \%$ respectively (Figure 1B). When T6VA and CM-T6VA were compared to fractions $10,30,100$ and $300 \mathrm{kDa}$, no statistical differences were found using a multiple comparison executed by the Bonferroni procedure (Figure 1B). No statistical difference was observed in blastocyst cell numbers between groups. As a consequence of the results with T6VA from the 2-cell stage we decided to test this same medium and fractions on

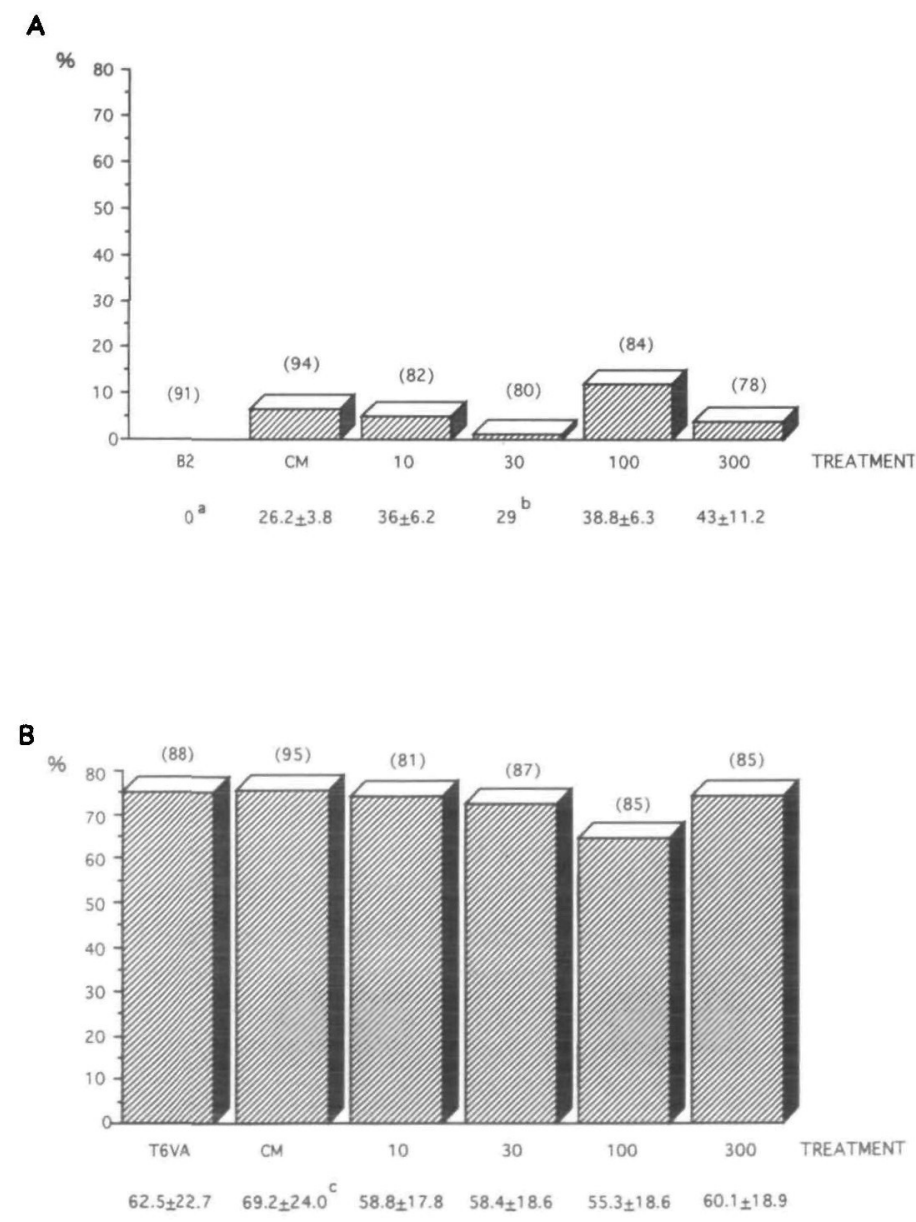

Figure 2. The percentage of 2-cell embryos developing to the blastocyst stage $120 \mathrm{~h}$ post-HCG on Vero cells (A) in B2, conditioned medium (CM-B2) and fractions $(<10,30,100$ and $300 \mathrm{kDa}$ ), and (B) in T6VA medium, conditioned medium (CM-T6VA) and fractions. Numbers of embryos observed are in parentheses. Mean cell numbers \pm SD of blastocysts are shown below the $x$ axes. (There was no significant difference in blastocyst development.) a'Embryos did not reach compacted or blastocyst stage; ${ }^{b}$ only one blastocyst was observed; ' ${ }^{\text {significantly different }}$ $(P<0.05)$ compared to mean cell numbers of embryos developed in the $100 \mathrm{kDa}$ fraction. 
embryos derived from IVF. As expected, embryos cultured after IVF were very sensitive to their environment. In three replicates, between 44 and $62 \%$ of embryos in each culture group had >4 cells (T6: 32/51; CM-T6: 19/43; fraction <10 kDa: 22/58; 30 kDa: 27/58; 100 kDa: 29/58; 300 kDa: 31/67). No significant difference was apparent in the ability of the different fractions to allow embryos to reach these stages. Most embryos stopped development at the morula stage; only 5.3 and $6.5 \%$ reached the blastocyst stage in CM-T6VA and fraction $300 \mathrm{kDa}$ respectively.

\section{Embryo development from the 2-cell stage in different media conditioned by Vero cells}

To examine the role of different cell types in coculture, we also assessed 2-cell embryo development on Vero cells.

\section{Ménézo's $B 2$}

Poor embryo development was observed to the blastocyst stage in all groups (B2: 0/91; CM-B2: 6/94; fraction <10 kDa: 4/84; $30 \mathrm{kDa}: 1 / 80$; $100 \mathrm{kDa}: 10 / 84 ; 300 \mathrm{kDa}: 3 / 78$ ) (Figure 2A). In contrast, control 2-cell embryos cultured in T6VA in parallel developed to blastocysts at a high rate $(46 / 60,76.7 \%)$. These experiments confirmed previous results in which coculture of 2-cell mouse embryos on Vero cells using B2 led to poor blastocyst development (8\%) (Ouhibi et al., 1990).

\section{T6VA medium}

All culture groups provided a high percentage of blastocysts, ranging from 64.7 to $75.8 \%$ at $120 \mathrm{~h}$ post-HCG (Figure 2B). There was no statistically significant difference in blastocyst formation between the different media tested. Cell numbers were high, the highest being observed in total conditioned medium (69.3 \pm 24.0$)$, in which blastocyst formation was also the highest (Figure 2B). This conditioned medium was statistically different in cell numbers when compared to the $100 \mathrm{kDa}$ fraction, but not to the other media tested. The mean cell number $( \pm S D)$ of in-vivo developed blastocysts recovered at $96 \mathrm{~h}$ post-HCG was $49.3 \pm 13.17$.

\section{Discussion}

A number of studies have shown a beneficial effect of coculture on embryo development. For example, Gandolfi and Moor (1987) showed that coculture with oviduct cells enhanced cleavage-stage sheep embryo development compared to culture medium without cells. A positive effect from coculture has also been shown by Ménézo et al. (1990) in human embryo development and by Ouhibi et al. (1990) and Lai et al. (1992) in mouse. At the same time, similar experiments using IVF bovine embryos in coculture with oviduct cells using TCM199 without FCS (Pinyopummintr and Bavister, 1991) failed to show any benefit from coculture. In addition to culturing embryos directly on top of cells, a number of groups have investigated the use of conditioned medium. Different studies reported improved cattle embryo development using medium conditioned by oviductal tissue (M199 with FCS medium; Eyestone and First, 1989; Mermillod et al., 1993) or granulosa cells (TCM199 without FCS; Kobayshi et al., 1992). Again, conflicting results were reported by Rexroad and Powell (1988), who could not show an enhancement in sheep embryo development in Ham's F-10 with FCS conditioned on oviductal cells. These conflicting results have led to questions concerning the real necessity of coculture (Bavister, 1992).

The disparity in the studies examining coculture and conditioned medium leads to an examination of the experimental systems. An initial problem may exist in the selection of the base medium. Medium in coculture has to support two systems: cells and embryo. Rexroad and Powell (1988) showed a dramatic effect of Ham's F-10 medium on cells (cell death), compared to a beneficial effect on the embryo. The converse observation with DMEM showed that the medium was beneficial for the cells but poor in supporting embryo development. Therefore, an extreme difficulty exists in finding a balanced medium, because as well as affecting the cells, the type of culture medium can also dramatically affect the embryo. Even media designed for improving embryo development can show vast differences in their effect on preimplantation embryos. For example, by changing the composition of medium, embryos can change their glycolytic activity. Gardner and Sakkas (1993) showed variation in blastocyst glycolytic activity when medium contained different combinations of amino acids, vitamins, insulin, epidermal growth factor and transferrin. In a separate study, Gardner and Lane (1993) found that production of ammonium ions from the breakdown of amino acids may also be toxic for the embryo, and they proposed that medium should be changed every $48 \mathrm{~h}$ in order to reduce this effect. This observation occurs when only embryos and medium have been in contact. By using cells, yet another variable is introduced which could lead to changes in the medium and affect the embryo. During coculture experiments, the cells, medium and embryo are in contact for several days; consequently there is a continuous change in the medium and the production of toxic factors is highly feasible.

The conflicting results from the studies performed using coculture also mean comparisons between different studies are difficult. In coculture with Vero cells, Ménézo et al. (1990) and Ouhibi et al. (1990) observed beneficial effects of Vero cells on coculture of human embryos but not with 1-cell mouse embryos. In contrast, Lai et al. (1992) reported mouse embryo development from the 2-cell stage until the blastocyst stage using Vero cells. These studies, however, cannot be compared directly as they used different media, different strains of mice and were started at different stages of embryo development. Sensitivity of different embryo stages is diverse; an embryo developed until the 2-cell stage in vivo is submitted to less stress than an oocyte fertilized in vitro. In our study, medium or conditioned medium (T6VA on MDBK) which was supporting blastocyst formation from the 2-cell stage lost all its beneficial effects when embryos were cultured from the IVF stage. This demonstrates the greater sensitivity of an embryo developed in vitro compared to in vivo. In-vivo embryo development occurs in an ever-changing environment and not in a static environment, as is used in many embryo culture studies. As previously suggested, medium composition should therefore be changed during embryo development (Brown and Whittingham, 1991; Gardner and Sakkas, 1993).

The ultimate goal of coculture studies is to find evidence of the presence of a factor or factors that enhances preimplantation 
embryo development. In the light of this, several studies have argued that a number of different cell lines may produce such a factor(s) (Ménézo et al., 1990; Ouhibi et al., 1990; Minami et al., 1992; Bongso et al., 1993; Mermillod et al., 1993). In this study only B2 medium conditioned by MDBK cells improved embryo development, but no blastocyst formation was observed in B2 alone. This suggests that the cells induced a modification of the base medium which gave a positive effect. In contrast, the benefits observed using MDBK cells were lost with Vero cells, suggesting either that some cell lines are unable to remove inhibitory factors from a poor medium such as B2 or that inhibiting factors are produced by certain cell lines. In addition, contrary to studies reporting the existence of a low molecular weight molecule which enhanced embryo development (Minami et al., 1992; Mermillod et al., 1993), no beneficial effect was consistently observed in any specific fraction tested in our work. This supports the idea that cells are responsive only to certain media and that feeder cells do not improve embryo development through specific factors secreted into the medium but only act by changing the base medium.

The interpretation of the action of the cells on the medium and their beneficial effect is still not clear. A more efficient means of demonstrating enhancement of embryo development could be to start from an original embryo culture medium and introduce individual factors derived from fractions of conditioned medium. An embryotrophic factor might be observed by starting from positive conditions. Consequently, coculture results have to be interpreted carefully, as the presence of many variables can lead to misinterpretation of results and false positives. For example, even the animal model used can be important. The mouse model used here might not be the most appropriate to show a beneficial effect since the effectiveness of the conditioned medium was poor for embryos cultured after IVF. The use of other animal models, such as bovine (Eyestone and First, 1989; Xu et al., 1992), could be more appropriate.

In conclusion, we have observed that media (B2 and T6VA) conditioned on MDBK and Vero cells gave vastly different responses in supporting embryo development. The lack of a strong positive effect on embryo development by the same fraction, regardless of media or cells, is not supportive of the presence of a specific embryo enhancing factor(s). In addition, when using Ménézo's B2 medium, no blastocyst formation was observed unless the medium had been conditioned by MDBK cells. The results therefore indicate that the positive effects reported in coculture and conditioned media studies are more likely governed by the choice of base medium and not by the presence of embryotrophic factors.

\section{Acknowledgements}

The authors thank Madame Bernadette Mermillod for statistical advice. This work was supported by a grant from the Fonds National Suisse (No. 32-30145.90) and the Sir Jules Thorn Charitable Overseas Trust Fund.

\section{References}

Bavister, B.D. (1992) Co-culture for embryo development: is it really necessary? Hum. Reprod., 7, 1339-1341.
Bongso, A., Ng, S.C., Sathananthan, H., Ng, P.L., Rauff, M. and Ratnam, S.S. (1989) Improved quality of human embryos when co-cultured with human ampullary cells. Hum. Reprod., 4, 706-713.

Bongso, A., Fong, C.-Y., Ng, S.-C. and Ratnam, S. (1993) The search for improved in-vitro systems should not be ignored: embryo coculture may be one of them. Hum. Reprod., 8, 1155-1162.

Brown, J.J.G. and Whittingham, D.G. (1991) The roles of pyruvate, lactate and glucose during preimplantation development of embryos from F1 hybrid mice in vitro. Development, 112, 99-105.

Chatot, C.L., Ziomek, C.A., Bavister, B.D., Lewis, J.L. and Torres, I. (1989) An improved culture medium supports development of random-bred 1-cell mouse embryos in vitro. J. Reprod. Fertil., 86, 679-688.

Ellington, J.E., Carney, E.W., Farrell, P.B., Simkin, M.E. and Foote, RH. (1990) Bovine 1-2-cell embryo development using a simple medium in three oviduct epithelial cell coculture systems. Biol. Reprod., 43, 97-104.

Eyestone, W.H. and First, N.L. (1989) Co-culture of early cattle embryos to the blastocyst stage with oviductal tissue or in conditioned medium. J. Reprod. Fertil., 85, 715-720.

Gandolfi, F. and Moor, R.M. (1987) Stimulation of early embryonic development in the sheep by co-culture with oviduct epithelial cells. J. Reprod. Fertil., 81, 23-28.

Gardner, D.K. and Lane, M. (1993) Amino acids and ammonium regulate mouse embryo development in culture. Biol. Reprod., 48, $377-385$

Gardner, D.K. and Sakkas, D. (1993) Mouse embryo cleavage, metabolism and viability: role of medium composition. Hum. Reprod., 8, 288-295.

Goddard, M.J. and Pratt, H.P. (1983) Control of events during early cleavage of the mouse embryo: an analysis of the '2-cell block'. J. Embryol. Exp. Morphol., 73, 111-133.

Harlow, G.M. and Quinn, P. (1982) Development of preimplantation mouse embryos in vitro and in vivo. Aust. J. Biol. Sci., 35, 187-193.

Kobayshi, K., Takagi, Y., Satoh, T., Hoshi, H. and Oikawa, T. (1992) Development of early bovine embryos to the blastocyst stage in serum-free conditioned medium from bovine granulosa cells. In Vitro Cell Dev. Biol., 28A, 255-259.

Lai, Y.M., Stein, D.E., Soong, Y.K., Tang, Y.X., Grifo, J., Malter, H.E., Talansky, B.E., Cohen, J., Liu, H.C. and Rosenwaks, Z. (1992) Evaluation of Vero cell co-culture system for mouse embryos in various media. Hum. Reprod., 7, 276-280.

Ménézo, Y., Guerin, J.F. and Czyba, J.C. (1990) Improvement of human early embryo development in vitro by co-culture on monolayers of Vero cells. Biol. Reprod., 42, 301-306.

Mermillod, P., Vansteenbrugge, A., Wills, C., Mourmeaux, J.-L., Massip, A. and Dessy, F. (1993) Characterisation of the embryotrophic activity of exogenous protein-free oviductconditioned medium used in culture of cattle embryos. Biol. Reprod., 49, 582-587.

Minami, N., Utsumi, K. and Iritani, A. (1992) Effects of low molecular weight oviductal factors on the development of mouse one-cell embryos in vitro. J. Reprod. Fertil., 96, 735-745.

Ouhibi, N., Hamidi, J., Guillaud, J. and Ménézo, Y. (1990) Co-culture of I-cell mouse embryos on different cell supports. Hum. Reprod., 5, 737-743.

Pinyopummintr, T. and Bavister, B.D. (1991) In vitro-matured/ in vitro-fertilised bovine oocytes can develop into morulae/ blastocysts in chemically defined, protein-free culture media. Biol. Reprod., 45, 736-742.

Quinn, P., Barros, C. and Whittingham, D.G. (1982) Preservation of hamster oocytes to assay the fertilising capacity of human spermatozoa. J. Reprod. Fertil.. 66, 161-168.

Rexroad, C.E. and Powell, A.M. (1988) Co-culture of ovine eggs with oviductal cells and trophoblastic vesicles. Theriogenology, 29, 387-397. 
Sakkas, D. and Trounson, A.O. (1990) Coculture of mouse embryos with oviduct and uterine cells prepared from mice at different days of pseudopregnancy. J. Reprod. Fertil., 90, 109-118.

Sakkas, D., Trounson, A.O. and Kola, I. (1989a) In vivo cleavage rates and viability obtained for early cleavage mouse embryos in co-culture with oviduct cells. Reprod. Fertil. Dev., 1, 127-136.

Sakkas, D., Batt, P.A. and Cameron, A.W.N. (1989b) Development of preimplantation goat (Capra hircus) embryos in vivo and in vitro. J. Reprod. Fertil., 87, 359-365.

Sakkas, D., Urner, F., Ménézo, Y. and Leppens, G. (1993) Effects of glucose and fructose on fertilisation, cleavage and viability of mouse embryos in vitro. Biol. Reprod., 49, 1288-1292.

Sakkas, D.. Jaquenoud, N., Leppens, G. and Campana, A. (1994) Comparison of results after in vitro fertilised human embryos are cultured in routine medium and in co-culture on Vero cells: a randomised study. Fertil. Steril., 61, 521-525.

Tarkowski, A.K. (1966) An air-drying method for chromosome preparations from mouse eggs. Cytogenetics, 5, 394-400.

Wiemer, K.E., Cohen, J., Wiker, S.R., Malter, H.E., Wright, G. and Godke, R.A. (1989) Coculture of human zygotes on fetal bovine uterine fibroblasts: embryonic morphology and implantation. Fertil. Steril., 52, 503-508.

Whittingham, D.G. (1971) Culture of mouse ova. J. Reprod. Fertil., 14 (Suppl.), 7-21.

Xu, K.P., Yadav, B.R., Roriet, R.W., Plante, L., Betteridge, K.J. and King, W.A. (1992) Development and viability of bovine embryos derived from oocytes matured and fertilized in vitro and co-cultured with bovine oviductal epithelial cells. J. Reprod. Fertil., 94, 33-43.

Yeung, W.S.B., Ho, P.C., Lau, E.Y.L. and Chan, S.T.H. (1992) Improved development of human embryos in vitro by a human oviductal cell co-culture system. Hum. Reprod., 8, 1144-1149.

Received on July 11, 1994; accepted on February 14, 1995 